\title{
Newborn Hearing Screening-Experience at a Tertiary Hospital in Northwest India
}

\author{
John Jewel $^{1}$, P. V. Varghese ${ }^{1}$, Tejinder Singh ${ }^{1}$, Ashish Varghese ${ }^{2}$ \\ ${ }^{1}$ Department of Paediatrics, Christian Medical College, Ludhiana, India \\ ${ }^{2}$ Department of ENT_-Head and Neck Surgery, Christian Medical College, Ludhiana, India \\ Email: ashishvargheseent@gmail.com
}

Received June 29, 2013; revised July 30, 2013; accepted August 9, 2013

Copyright (C) 2013 John Jewel et al. This is an open access article distributed under the Creative Commons Attribution License, which permits unrestricted use, distribution, and reproduction in any medium, provided the original work is properly cited.

\begin{abstract}
Objective: To determine the incidence of hearing impairment in a standardized population of neonates seeking care in a tertiary hospital in Northwest India. Universal hearing screening is implemented in many developed countries. However, neither universal screening, nor high risk screening, exists in India. The incidence of hearing loss in India is found to be 1 to 6 per 1000 newborns screened [1-3]. Screening only the high risk neonates misses $50 \%$ of babies with hearing loss [4,5], hence a cost effective universal screening is the viable option to sustain such a program. In our study, the possible burden of hearing disability was evaluated in babies born at a tertiary care hospital in Northwest India. One thousand newborns were screened using Transient Evoked OtoAcoustic Emissions (TEOAE) and 28.6\% of them had risk factors. Four out of One Thousand were detected with hearing loss. Brain Stem Evoked Response (BERA) was used to confirm and determine the extent and the type of deafness in the neonates who were screened positive.
\end{abstract}

Keywords: Hearing Loss; Newborns; Transient Evoked OtoAcoustic Emissions; Universal Screening; Brainstem Evoked Response Audiometry (BERA)

\section{Introduction}

Hearing is a vital part of a newborn's contact with his environment. The ability to communicate, acquire skills, and perform academically is all greatly dependent on the ability to hear; especially in the present era which is quite dependent on audio-video based on technology. The less privileged youth of our country depends largely on business outsourced from other countries for economic stability. In this scenario, hearing and language skills are of prime importance, even to the poor urban slum dweller.

As hearing impairment is a hidden disability, it is usually detected after 2 years, by which time there is irreversible stunting of the language development potential [4]. Many developed countries have well established universal neonatal hearing screening programs. Considering the infrastructure limitations in India, it is crucial to adopt a cost effective way of detecting hearing loss to make this program viable. This study was undertaken to evaluate the possible burden of hearing loss among the neonates born in a tertiary care center in northern India and to justify the implementation of a universal hearing screening program in India, using cost effective and appropriate technology. Screening of neonates was done using Transient Evoked OtoAcoustic Emissions (TEOAE) and Automated Auditory Brainstem Response (ABR).

This study was undertaken in order to detect the frequency of congenital hearing loss among neonates in a tertiary care center in North India. The study also identifies the challenges in implementing a universal screening programme in normal neonates in North India and is among the few similar articles from North India.

\section{Material and Methods}

The study was conducted prospectively on all neonates born in Christian Medical College and Hospital, Ludhiana from 1st August, 2007 to 31st January, 2009.

Parents or the grandparents of the neonates were informed about the study and motivated to undergo the screening program. An informed consent was taken from the parent/guardian and approval of research and ethics committee was obtained.

Using a pretested questionnaire [6], potential risk factors were identified. Both the normal and high-risk neonates underwent hearing assessment after 48 hours of birth using TOAE as the first level of screening. Neonates who failed the initial screening were subjected to 
repeat testing with TOAE after one month (Screening Algorithm, Table 1). This was done in the Department of Otolaryngology at Christian Medical College and Hospital, Ludhiana using a GSI Audio Screener SN20008P ${ }^{\mathrm{TM}}$, which is a completely automated analysis system that gives a "PASS" or "REFER" result. Absence of emissions for 2 out of the 3 frequencies tested ( $2 \mathrm{kHz}, 3 \mathrm{kHz}$ and $4 \mathrm{kHz}$ ) was given a "REFER" result. Infants who failed the screening twice were referred to the Audiologist (Table 1).

Data from the questionnaire and the results of the testing were tabulated in Microsoft EXCEL ${ }^{\mathrm{TM}}$ and subjected to analysis using student $t$-test and coefficient of correlation.

\section{Results}

Among the 1000 neonates that were screened initially, 60 babies failed the first screening (6\%).

Forty two out of the failed neonates came for follow up, out of which 4 babies failed in the second screening as well. Hearing loss in these 4 babies was confirmed using ABR.

Three neonates out of the 4 who failed to have identifiable risk factors, which were low birth weight $<1.5 \mathrm{~kg}$ (1 baby), severe birth asphyxia (1), NNH requiring exchange blood transfusion (1), and meningitis (1). 1 baby had no risk factor for hearing loss.

The babies who were screened positive for hearing loss were confirmed using ABR. Two of them had severe Sensorineural hearing losses and the other two were diagnosed with moderate to severe hearing loss. All the babies were referred to an Audiologist for further interventions (Table 2).

\section{Discussion}

It is well recognized that unidentified hearing loss can adversely affect optimal speech and language development, acquisition of literacy skills, academic, social and emotional development. There is robust evidence that the identification and remediation of hearing loss, when done before six months of age for newborn infants who are hard of hearing, enable them to perform significantly higher on vocabulary, communication, intelligence, social skills and behavior necessary for success in later life [4]. In 1994, the Joint Committee on Infant Hearing (JCIH) established in the United States recommended screening of high risk babies for hearing loss using High Risk Registry [7]. Several studies thereafter suggested that up to $50 \%$ of all the children with congenital hearing loss have no risk factors and would be missed by screening only those at high risk [8-11]. American Academy of Pediatrics (AAP) in 1999 advocated universal newborn hearing screening programme (UNHSP) and remedial
Table 1. Screening algorithm.

\begin{tabular}{cccc}
\hline & Total Neonates & Test Passed & Failed Test \\
\cline { 2 - 4 } Initial screening & 1000 & 940 & 60 \\
\hline $\begin{array}{c}\text { Second screening } \\
\text { (18 lost to follow up) }\end{array}$ & 42 & 38 & 4 \\
\hline
\end{tabular}

Confirmation with ABR: Moderate to profound sensorineural hearing loss4; At risk infants-3; Neonate with no identifiable risk factor-1.

Table 2. Distribution of cases according to risk factors for hearing loss.

\begin{tabular}{cccc}
\hline & PASS & FAIL & TOTAL \\
\hline Birth weight less than 1.5 kg & 35 & 1 & 36 \\
Asphyxia & 4 & 1 & 5 \\
Family history of hearing impairment & 5 & 0 & 5 \\
NNH Requiring Exchange Transfusion & 52 & 1 & 53 \\
Meningitis & 1 & 1 & 2 \\
At risk neonates & 260 & 3 & 263 \\
Neonates with no risk factors & 736 & 1 & 737 \\
\hline
\end{tabular}

intervention, which is being practiced in most of the developed countries. The AAP Task Force on newborn and infant hearing recommends UNHS by three months of age with intervention by six months of age. The Joint Committee on Infant Hearing (JCIH) position statement provides guidelines that include Newborn Hearing Screening (NHS) soon after birth, before discharge from hospital, or before one month of age, diagnosis of hearing loss through audiological and medical evaluation before three months, and intervention through interdisciplinary programs for infants with confirmed hearing loss before six months of age [6].

\subsection{Rationale for Universal Hearing Screening in Newborns}

Universal screening for hearing loss is based on the following concepts:

1) It is seen that $42 \%$ - $70 \%$ of children will be missed using only risk-based screening [5,12].

2) A critical period exists for optimal language development and earlier interventions may produce better results.

3) Treatment of hearing defects has been shown to improve communication, better self-confidence and alleviate parental frustration and guilt.

Over the study period from 1st August, 2007 till 31st January 2009, we screened a total of 1000 babies and the incidence of hearing loss as per our observation is 4 per 1000 babies tested, out of which 3 babies had high risk factors for hearing loss and 1 baby was a well baby (Table 1). 


\subsection{TEOAE vs BERA as Initial Screening Method}

Otoacoustic emissions are the most sensitive tests for screening although it may have to be combined with other tests for complete diagnosis [13]. However it may give false results in the presence of debris or vernix in the external auditory canal of newborn babies. Brainstem Evoked Response Audiometry (BERA), though highly reliable, requires high technical expertise, which is more expensive as opposed to TEOAE. Also BERA makes use of a cumbersome machine whilst the TEOAE screener is a portable machine. It also requires sedating the infant because of the lengthy procedure. TEOAE, on the other hand does not assess the cortical pathway for hearing. It may also give false results in neonates with auditory neuropathy. However, it is an excellent tool as an initial screening method.

The relative advantages and disadvantages of a twostage (OAE/BERA) protocol for newborn hearing screening need to be considered carefully for individual circumstances. Transient Evoked OtoAcoustic Emissions (TEOAE) are a non-invasive and inexpensive test that can be done in the nursery setting with little expertise and a shorter time as compared to BERA. Different studies have revealed TEOAE sensitivity as high as 95\% $98 \%$ and a specificity of $80 \%-85 \%[14,15]$. Therefore, TEOAE cannot completely replace BERA as a screening modality, but can only complement it. In locations where getting infants to return for outpatient screening and testing is very difficult, and the substantially lower failure rate that will likely be achieved by using both OAE and BERA at the same sitting has significant advantages. In a setting like ours, this may not be very practical, but has to be considered wherever possible.

\subsection{Problems and Limitations of Study}

One problem we faced was getting a noiseless surrounding in the nursery setting. The babies had hence to be transported to the audiology room for testing which increased the discomfort for the relatives. Some babies woke up during transit, increasing the time taken for the test.

To improve the follow-up rate, we coincided the immunization visit with that of screening. Performing a test on that day was a little time consuming because one has to wait for the baby to go to natural sleep.

A hearing screening equipment facility in every hospital with a maternity unit today may not be an economically viable proposition. In this background, a practical interventional model was conceived in the city of Cochin (which has 20 hospitals with maternity units) in January 2003. A program with centralized screening facility, where a screener would operate out of one hospital, to cater to the different hospitals of the city was successfully implemented with the co-operation of IAP [16]. This is a viable and cost effective model for the whole country.

\section{REFERENCES}

[1] K. R. Bachmann and J. V. Arvedson, "Early Identification and Intervention for Children Who Are Hearing Impaired,” Pediatrics in Review, Vol. 19, 1998, pp. 155-165.

[2] P. Nagapoornima, A. Ramesh, Srilakshmi, S. Rao, P. L. Patricia, M. Gore, M. Dominic and Swarnarekha, "Universal Hearing Screening,” Indian Journal of Pediatrics, Vol. 74, No. 6, 2007, pp. 545-549. doi:10.1007/s12098-007-0105-z

[3] Rehabilitation Council of India, "Status of Disability in India-2000,” New Delhi, 2000, pp. 172-185.

[4] Yoshinaga, S. Itano, A. L. Sedey, D. K. Coulter and A. L. Mehl, "Language of Early and Later Identified Children with Hearing Loss,” Pediatrics, Vol. 102, No. 5, 1998, pp. 1161-1171. doi:10.1542/peds.102.5.1161

[5] J. Hadad Jr., "Hearing Loss,” In: R. E. Behrman, R. Kliegman and H. B. Jenson, Eds., Nelson Textbook of Pediatrics, 17th Edition, Saunders, Philadelphia, 2004, pp. 21292134.

[6] Joint Committee on Infant Hearing, American Academy of Audiology, American Academy of Pediatrics, American Speech-Language-Hearing Association, Directors of Speech and Hearing Programmes in State Health and Welfare Agencies, "Year 2000 Position Statement: Principles and Guidelines for Early Hearing Detection and Intervention Programmes,” Pediatrics, Vol. 106, 2000, pp. 798-817.

[7] Joint Committee on Infant Hearing, "Joint Committee on Infant Hearing (JICH) 1994 Position Statement," Pediatrics, Vol. 95, 1994, pp. 152-156.

[8] M. A. M. De, “Newborn Hearing Screening," eMedicine, 2004, pp. 1-14.

[9] K. R. White, B. R. Vohr and T. R. Behrens, "Universal Newborn Hearing Screening Using Transient Evoked Otoacoustic Emissions: Results of the Rhode Island Hearing Assessment Project," Seminars in Hearing, Vol. 14, 1993, pp. 18-29.

[10] G. Isaacson, "Universal Newborn Hearing Screening and Intervention,” Advances in Otolaryngology-Head and Neck Surgery, Vol. 15, 2001, pp. 1-19.

[11] K. White and A. Maxon, "Universal Screening for the Hearing Impairment: Simple, Beneficial and Presently Justified," International Journal of Pediatric Otorhinolaryngology, Vol. 32, No. 3, 1995, pp. 201-211. doi:10.1016/0165-5876(95)01165-8

[12] www.nnfpublication.org

[13] K. L. Stein, "Factors Influencing the Efficacy of Universal Newborn Hearing Screening," Pediatric Clinics of North America, Vol. 46, No. 1, 1996, pp. 95-102. doi:10.1016/S0031-3955(05)70084-5

[14] S. J. Norton, M. P. Gorga, J. E. Widen, R. C. Folsom, Y. 
Sinnger, B. Cone-Wesson, et al., "Identification of Neonatal Hearing Impairment: Evaluation of Transient Evoked Otoacoustic Emission, Distortion Product Otoacoustic Emission, and Auditory Brainstem Response Test Performance,” Ear and Hearing, Vol. 21, 2000, pp. 508-528. doi:10.1097/00003446-200010000-00013
[15] R. Keren, M. Helfand, C. Homer, H. McPhilips and T. A. Lieu, "Projected Cost Effectiveness of Statewide Universal Newborn Hearing Screening,” Pediatrics, Vol. 110, No. 5, 2002, pp. 855-864. doi:10.1542/peds.110.5.855

[16] K. P. Abraham, "Newborn Hearing Screening," Indian Pediatrics, Vol. 48, No. 5, 2011, pp. 355-359. 